\title{
Bertrand QUENTIN
}

Agrégé et docteur en philosophie, Professeur en classes préparatoires à Claude-Bernard (Paris XVIe)

(2008)

\section{"Hegel était-il perméable aux totalitarismes?"}

Un document produit en version numérique par Jean-Marie Tremblay, bénévole, professeur de sociologie au Cégep de Chicoutimi

Courriel: jean-marie tremblay@uqac.ca

Site web pédagogique : http://www.uqac.ca/jmt-sociologue/

Dans le cadre de la collection: "Les classiques des sciences sociales" Site web: http://classiques.uqac.ca/

Une collection développée en collaboration avec la Bibliothèque Paul-Émile-Boulet de l'Université du Québec à Chicoutimi

Site web: http://bibliotheque.uqac.ca/ 


\section{Politique d'utilisation de la bibliothèque des Classiques}

Toute reproduction et rediffusion de nos fichiers est interdite, même avec la mention de leur provenance, sans l'autorisation formelle, écrite, du fondateur des Classiques des sciences sociales, Jean-Marie Tremblay, sociologue.

Les fichiers des Classiques des sciences sociales ne peuvent sans autorisation formelle:

- être hébergés (en fichier ou page web, en totalité ou en partie) sur un serveur autre que celui des Classiques.

- servir de base de travail à un autre fichier modifié ensuite par tout autre moyen (couleur, police, mise en page, extraits, support, etc...),

Les fichiers (.html, .doc, .pdf., .rtf, .jpg, .gif) disponibles sur le site Les Classiques des sciences sociales sont la propriété des Classiques des sciences sociales, un organisme à but non lucratif composé exclusivement de bénévoles.

Ils sont disponibles pour une utilisation intellectuelle et personnelle et, en aucun cas, commerciale. Toute utilisation à des fins commerciales des fichiers sur ce site est strictement interdite et toute rediffusion est également strictement interdite.

L'accès à notre travail est libre et gratuit à tous les utilisateurs. C'est notre mission.

Jean-Marie Tremblay, sociologue

Fondateur et Président-directeur général, LES CLASSIQUES DES SCIENCES SOCIALES. 
Cette édition électronique a été réalisée par Jean-Marie Tremblay, bénévole, professeur de sociologie au Cégep de Chicoutimi à partir de :

\section{Bertrand QUENTIN}

\section{“Hegel était-il perméable aux totalitarismes ?”}

Un article publié dans la revue CAUSE COMMUNE, revue citoyenne d'actualité réfléchie, 2008. Paris : Éditions du Cerf.

L’auteur, Bertrand Quentin, Agrégé et docteur en philosophie, Professeur en classes préparatoires à Claude-Bernard (Paris XVIe), nous a accordé le 9 janvier 2008 son autorisation de diffuser électroniquement ce texte dans Les Classiques des sciences sociales.

Le directeur de la revue, M. Pierre Dupuis, nous a également donné son autorisation de diffuser cet article dans Les Classiques des sciences sociales.

Courriel : Bertrand.Quentin@ac-paris.fr

Polices de caractères utilisée :

Pour le texte: Times New Roman, 12 points.

Pour les citations : Times New Roman, 12 points.

Pour les notes de bas de page : Times New Roman, 12 points.

Édition électronique réalisée avec le traitement de textes Microsoft Word 2004 pour Macintosh.

Mise en page sur papier format : LETTRE (US letter), 8.5'” x 11'’)

Édition numérique réalisée le 10 janvier 2009 à Chicoutimi, Ville de Saguenay, province de Québec, Canada. 
Nous voulons remercier le directeur de la revue CAUSE COMMUNE, M. Pierre Dupuis, de nous avoir donné son autorisation, conjointement avec celle de l'auteur, M. Bertrand Quentin, de diffuser le texte intégral de cet article, "Hegel étaitil perméable aux totalitarismes ?”

Merci de votre confiance en nous et longue vie à la revue CAUSE COMMUNE.

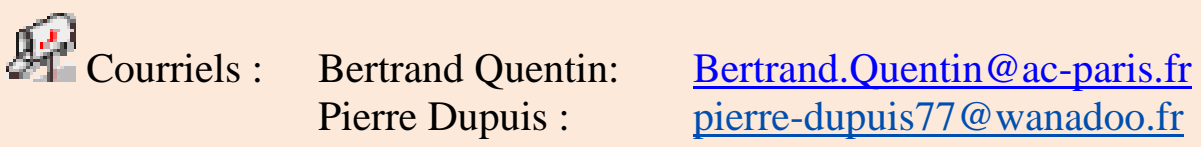

Merci,

Jean-Marie Tremblay, sociologue.

Fondateur et PDG, Les Classiques des sciences sociales.

10 janvier 2009. 
L'auteur a publié en 2008, chez L'Harmattan, le livre : HEGEL ET LE SCEPTICISME. Collection : Ouverture philosophique, 315 pp.

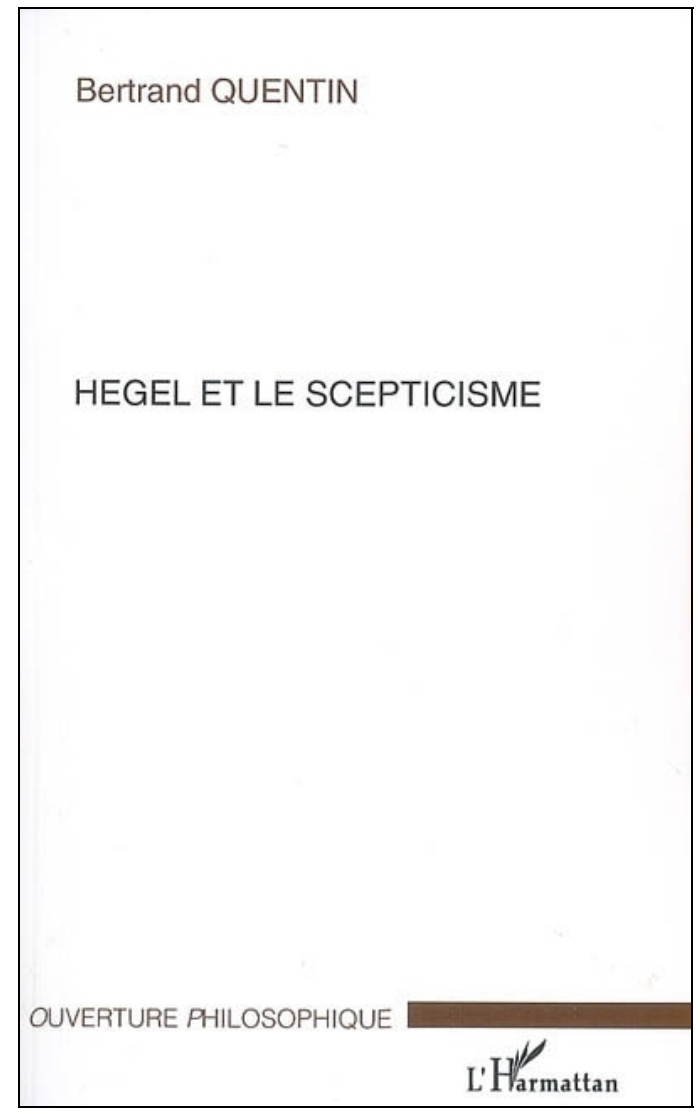

"Hegel a, dans l'opinion, une réputation de dogmatisme. Le présent ouvrage vise à montrer au contraire que, dans sa confrontation au scepticisme, le penseur allemand manifeste une ouverture philosophique étonnante et inédite : le scepticisme ne sera pas représenté comme un "ennemi à abattre" mais comme un moment incontournable de toute pensée. Il faudra ainsi passer par lui pour accéder au Système de la Science.” (L’éditeur) 


\section{Table des matières}

$\underline{\text { Introduction }}$

1) Le particulier est reconnu par Hegel, mais ne pourra être repris spéculativement.

2) Il y a pour Hegel un Mal radical (il ne peut, en tant que tel, être repris).

3) À l'encontre de tout relativisme conformiste, l'hégélianisme met en place des critères permettant de distinguer un bien et un mal.

4) Hegel a en réalité indiqué certaines sources du Mal totalitaire. 


\section{Bertrand QUENTIN}

“Hegel était-il perméable aux totalitarismes ?”

Un article publié dans la revue CAUSE COMMUNE, revue citoyenne d'actualité réfléchie, 2008. Paris : Éditions du Cerf.

\section{Introduction}

$\underline{\text { Retour à la table des matières }}$

Hegel a à l'esprit des images fortes de réconciliation du particulier et de l'universel au niveau politique. La Cité grecque a longtemps représenté pour lui la « belle totalité » qu'il faut arriver à retrouver à travers le concept moderne d'Etat. L'individu s'accomplit ainsi de la manière la plus élevée dans une communauté forte. Mais du constat de l'insuffisance de l'individu, à l'allégeance totale à la réalité collective, il n'y a qu'un pas qu'une certaine interprétation du hégélianisme peut faire. B.Gilson nous dit par exemple : « (Hegel) affaiblit les éléments, déjà insuffisants, d'individualisme rationaliste auxquels Fichte reste fidèle. Il critique les lois tracassières, mais semble donner tort d'avance à toutes les protestations impuissantes. Qu'arrive-t-il si la «belle société » n’est pas belle ? Qu’arrive-t-il si elle étouffe la liberté au lieu de la favoriser ? Le hégélianisme politique consiste-til à tout accepter ? ». ${ }^{1}$ Hegel va être ainsi accusé de ne pouvoir nous offrir en politique autre chose qu'un conformisme qui épouse les méandres de l'histoire sans pouvoir jamais prendre le parti de l'individu. B. Bourgeois se sent ainsi obligé de concéder la remarque suivante : « Certes, dans son contenu total, la spé-

1 B.GILSON Présentation de la différence entre les systèmes philosophiques de Fichte et de Schelling de Hegel, Paris, Vrin, 1986. p.69. 
culation hégélienne n’érige pas la liberté humaine en principe absolu de l'être ». 2 À ce propos, Hegel a, il est vrai, des formules sans ambiguïté pour l’individu :

« Toute grandeur dans le monde a au-dessus d'elle-même quelque chose de plus élevé : voilà ce qu'il ne faut jamais perdre de vue. Le Droit de l’Esprit du Monde dépasse toutes les justifications particulières $» .3$

Une atmosphère inquiétante semble soudain prendre à la gorge le lecteur. Un «Esprit du Monde » mystérieux aurait le droit d’écraser l’individu ? Mais comment se définit cet «Esprit du Monde » qui surplomberait de façon inquiétante l'individu ? Kojève, dans son cours sur Hegel, précise : « comprendre l’Histoire comme le devenir de la Vérité - c’est l’hégélianisme. Les autres se trompent en croyant qu'ils peuvent par la seule raison parvenir à la connaissance de la vérité ». ${ }^{4}$ L’Histoire répondra donc pour nous. Une analyse de raison sera toujours insuffisante car en matière de vérité l'individu sera toujours un infirme. Comment, dès lors, mettre un frein au mouvement qui s'accusera dans le marxisme ? Marx nous expliquera que même la classe qui a pour objectif de supprimer tout rapport de domination entre les individus (le prolétariat) est obligée pendant un certain temps de maintenir une domination violente contre les individus par l'intermédiaire du Parti. Cette domination violente est justifiée comme étant de l'ordre de l'urgence. Le problème qui se pose alors est de savoir quand l'urgence doit cesser ? On en arrive aux dérives de Lénine et surtout de Staline dont les discours marxistes masquent un passage à une domination et à un climat de terreur politique permanents. La tendance à délégitimer toute analyse rationnelle de l'individu apparaît nettement dans le discours d'un Trotski : « Nous ne pouvons avoir raison qu'avec et par le Parti, car l'histoire n’a pas fourni d'autre moyen d'avoir raison. Les anglais ont un dicton, « Tort ou raison, c’est mon pays » (...) Nous avons une bien meilleure justification historique en disant : qu'il ait raison

2 B. BOURGEOIS, « Histoire et géographie : le destin de l'inhumain selon Hegel » ; conf. à Basse-Terre en 1989 ; in Etudes hégéliennes, Paris, PUF, 1992 ; p.251.

3 HEGEL, Cours donnés à Berlin (1822, 1828, 1830), die Vernunft in der Geschichte, ed. par Hoffmeister, Hamburg, F.Meiner Verlag, 1955 ; trad : La raison dans l'histoire, par K.Papaioannou, Paris, Plon, 1965, p.133/134.

4 A. KOJEVE, Introduction à la lecture de Hegel, Paris, Gallimard, 1947 ; p.249. 
ou qu'il ait tort dans certains cas concrets et individuels, c'est mon parti ». ${ }^{5}$ Le « Parti » remplace ici pour un temps l' « Esprit du Monde » hégélien mais on repère très bien la filiation qui d’un déni de l'autosuffisance de l'individu rationnel passe à une allégeance à une réalité collective qu’on ne se donne plus le droit individuel de questionner.

La manière hégélienne de philosopher contiendrait également en germe ce que Popper appelle des « stratégies auto-immunisantes ». ${ }^{6}$ Lorsque Hegel nous dit :

« la profondeur se mesure à la grandeur de l'opposition ». ${ }^{7}$

Plus une difficulté apparente semble grande, plus elle nous permet de faire un progrès considérable. Hannah Arendt, dans Le Système totalitaire, illustre alors concrètement l'application à un contexte politique de cet état d'esprit : « la dialectique hégélienne fournit un merveilleux instrument pour avoir toujours raison, car elle permet d'interpréter toutes les défaites comme le commencement de la victoire. L’un des plus beaux exemples de ce genre de sophisme fut fourni après 1933, lorsque les communistes allemands, pendant près de deux ans, refusèrent d'admettre que la victoire de Hitler avait été une défaite pour le parti communiste allemand ». ${ }^{8} \mathrm{H}$. Arendt parle de la dialectique hégélienne utilisée comme un instrument. Mais il peut y avoir un écart considérable entre l'état d'esprit de Hegel dans son travail philosophique et la façon dont d'autres penseurs (Marx compris) vont se saisir de ce qu’ils voient comme la « méthode hégélienne ».

5 TROTSKI cité par B.SOUVARINE Staline, Aperçu historique du bolchevisme, Paris, 1935 ; p.361.

6 Karl POPPER, Conjectures et réfutations, Paris, Payot, 1985.

7 HEGEL, Cours de Berlin, années 1820, Vorlesungen über die Geschichte der Philosophie, publié en 1833 par K.L.Michelet ; trad. Par P. Garniron Leçons sur l'histoire de la philosophie T7 (La philosophie moderne), Paris, Vrin, 1991, (noté Leçons-hist-philo T7), « Résultat », p.2111 ; G19, p.684.

8 H.ARENDT, Le système totalitaire, Paris, Le Seuil, 1972 ; Note 22 p.256. 


\section{Manières hégéliennes de répondre à ces inquiétudes.}

\section{1) Le particulier est reconnu par Hegel, mais ne pourra être repris spéculativement.}

$\underline{\text { Retour à la table des matières }}$

Loin d'avoir en tête un Absolu qui rende le particulier sans intérêt, il faut souligner fortement que Hegel reconnaît clairement le particulier et sa nécessité d'être en tant que particulier. C’est la définition même du fini qui est ici en jeu. Comme Hegel le dira en 1821 dans les Principes de la Philosophie du droit :

« Ce qui fait partie de la nature de la contingence rencontre toujours le contingent (...) Il est nécessaire que le fini - la vie et la propriété - soit posé comme contingent, parce que c'est cela qui constitue le concept du fini ».

Le concret (au sens courant) de la vie de tous les jours est tissé de sentiments et la forme de tout sentiment tient d'une certaine contingence :

« La forme du sentiment est qu’il est, certes, une affection déterminée, mais que cette déterminité est simple. C'est pourquoi un sentiment, même quand son contenu est le plus solide et le plus vrai, a la forme d'une particularité contingente, - sans compter que le contenu peut être aussi bien le plus indigent et le moins vrai ». 9

Il n’y a pas pour autant à désavouer ce particulier de la vie. Même pour le travail philosophique il faudra passer par le HIC ET NUNC de l'expérience :

« Le principe de l'expérience contient la détermination infiniment importante, que pour admettre et tenir pour vrai un contenu, l'homme doit lui-même y être présent (dabei sein), de façon plus précise, qu’il a

9 HEGEL, Enzyklopädie der philosophischen Wissenschaften (1827-1830) / éd. de F. Nicolin et O. Pöggeler - citée : NP /Encyclopédie des sciences philosophiques : III La Philosophie de l’Esprit, (noté désormais: EncycloEsprit) texte intégral présenté, traduit et annoté par Bernard BOURGEOIS, Paris, Vrin, 1988. (1827) \# 447 ; p.243 ; NP, p.361. 
à trouver un tel contenu en accord avec la certitude (Gewissheit) de lui-même et réuni avec elle. Il doit y être présent, soit seulement avec ses sens extérieurs, ou bien avec son esprit plus profond, sa conscience de soi essentielle ». 10

Hegel reconnaît donc bien cette contingence du particulier comme particulier. En même temps il y a un moment du travail philosophique où ce particulier doit en raison même de son caractère particulier être laissé à la vie HIC ET NUNC pour que le regard voit autre chose de l'histoire. Hegel dira donc que

« ce qui relève seulement de la sentimentalité a un autre sol et intérêt que l'histoire » 11 ou que « les particularités des individus sont au plus loin de cet ob-jet qui relève de l'histoire». 12

Cela l'amènera à faire la leçon aux philosophes du passé qui se sont, eux, laissés aller à un rationalisme outrancier au point d'oublier cette nécessité pour la raison de laisser la contingence à la contingence :

« Le rationnel est le synonyme de l’Idée. Mais, lorsque, avec son actualisation il entre aussi dans l'existence extérieure, il y apparaît sous une richesse infinie de formes, de phénomènes, de figures (...) Les circonstances infiniment diverses qui se forment dans cette extériorité par l'apparition de l'essence en elle, ce matériel infini et son système de régulation, ne constituent pas l'objet de la philosophie. Elle peut s’épargner la peine de donner de bons conseils en ce domaine. C'est ainsi que, par exemple, Platon, aurait pu s'abstenir de recommander aux nourrices de ne jamais laisser les enfants sans mouvement, de les bercer dans leurs bras, et Fichte de perfectionner la police des passeports, au point de suggérer qu'on ne fasse pas seulement figurer dans ces documents le signalement des suspects, mais encore leur portrait. Dans de telles déclarations, il n’y a plus la moindre trace de philosophie, et celle-ci peut d'autant plus négliger une sagesse excessive en ce domaine, qu'elle doit se montrer la plus libérale possible à l'égard de cette multitude de détails». 13

10 HEGEL, Enzyklopädie der philosophischen Wissenschaften (1827) / éd. de F. Nicolin et O. Pöggeler - citée : NP /Encyclopédie des sciences philosophiques : I La science de la logique, (noté désormais : Encyclo-Logiq) texte intégral présenté, traduit et annoté par Bernard BOURGEOIS, Paris, Vrin, 1986, \# 7 ; p.171 ; NP, p.40.

11 HEGEL, Encyclo-Esprit (1830), \# 549 ; p.330 ; NP, 429.

12 HEGEL, Encyclo-Esprit (1827), \# 549 ; p.329 ; NP, 428.

13 HEGEL Naturrecht und Staatswissenschaft im Grundrisse / Grundlinien der Philosophie des Rechts, Berlin, In der Nicolaischen Buchhandlung, 1821. 
Si tous ces exemples semblent encore «bon enfant » et si le particulier doit être reconnu d’une façon générale comme contingence de la vie, il y a des moments où le particulier est le lieu d'une souffrance physique ou psychologique où domine le sentiment d'injustice - en d'autre terme le lieu du Mal.

\section{2) Il y a pour Hegel un Mal radical (il ne peut, en tant que tel, être repris).}

\section{$\underline{\text { Retour à la table des matières }}$}

Hegel, lorsqu'il regarde l'histoire, n'a rien d'un optimiste rationaliste béat. Il affirme clairement qu'en tant que sujets individuels, le lot des hommes est de vivre « la croix du présent ». ${ }^{14}$ Dire qu’il y a des réalités qui semblent rebelles au concept (l’immédiateté du vécu, la réalité sordide de la violence) n’est pas lutter contre Hegel. C’est chercher avec Hegel (et contre Spinoza) à ne pas faire du vécu une simple illusion, lui redonner son « poids de chair », tout en reconnaissant que ce « poids » peut être vécu mais ne fait pas l’objet possible de la philosophie :

«Lorsqu'on voit les faits particuliers, on peut se dire qu'il y a bien des choses injustes dans le monde. Il y aurait donc beaucoup à redire en ce qui concerne les aspects individuels du monde phénoménal. Mais il ne s’agit pas ici des particularités empiriques : elles sont sujettes au hasard et ne nous concernent point ». 15

Ainsi, la pensée hégélienne ne nous mène pas à estimer que la mort de l'homme d'Auschwitz a «servi » la raison. La mort de cet homme ne peut être « reprise » par la raison. Auschwitz ne peut être « repris ». Auschwitz ne peut être

(porte comme année de publication 1821, mais a paru en octobre 1820 (la préface est datée du 25 juin 1820)) Hoffmeister, Hambourg, F.Meiner, 1955 ; trad : Principes de la philosophie du droit ou Droit naturel et science de l'Etat en abrégé (noté Principes-philo-droit) présenté, traduit et annoté par Robert DERATHE, Paris, Vrin, 1993, Préface, Hoffmeister, p.15 ; trad. p.56.

14 HEGEL, Principes-philo-droit, Préface, p.58.

15 HEGEL, Commentaire oral à La raison dans l'histoire, p.99. 
l'illustration du fameux thème hégélien de la «ruse de la raison » ${ }^{16}$. Dans son monstrueux non-sens le système concentrationnaire ne témoigne que du fait d'une ou de plusieurs volontés ivres d'elles-mêmes, ivres devant leur arbitraire possible, volontés relayées par une multitudes de vouloirs qui évitèrent la pensée au sein d'une organisation qui les y incita. Ce qui pourrait être « repris » dans une philosophie de l'histoire hégélienne c'est par exemple l'humiliation excessive du peuple allemand par les pays alliés en 1918 : en bonne dialectique hégélienne, les humiliations excessives se renversent en orgueil excessif.

B. Bourgeois a insisté clairement sur la reconnaissance par Hegel d'une contingence radicale également dans le monde de l'esprit : «Le hégélianisme reconnaît, dans le réel ou dans l'expérience, l'existence nécessaire de ce qui est proprement et abstraitement empirique, c'est-à-dire, en son contenu même, irrationnel.- Il y a dans la nature - et donc dans l'esprit en tant que, nature se niant, il est conditionné par elle, en particulier dans l'histoire - de l'irrationnel, de l'insignifiant, du monstrueux, du scandaleux, bref, du Mal. Hegel a toujours admis celuici, et comme Mal que nul optimisme ne saurait transformer en Bien. Il y a donc, pour lui, un irrationnel absolu en son contenu". ${ }^{17}$ Ainsi le tableau hégélien de l'histoire du monde n'est pas celui d'un progrès universel : il y a des périodes de « saccage » où les phénomènes empiriques ne sont pas ou peuvent à peine être « repris » par la raison :

« Il y a dans l’histoire mondiale plusieurs grandes périodes du développement qui sont passées sans paraître avoir eu de prolongement, (et) à la suite desquelles, bien plutôt, tout l'énorme acquis a été anéanti, et (de telle sorte qu')il a fallu malheureusement reprendre les choses par le début, pour parvenir de nouveau - grâce au faible secours éventuellement à attendre de débris, sauvés, des trésors d'une telle culture, et avec une dépense nouvelle immense de forces et de temps, de cri-

16 Nous rappelons que la notion de « ruse de la raison » réside dans le pouvoir qu'a la raison de mobiliser en elle-même son autre, c'est-à-dire la nature contre elle-même. " Ce fait que le but subjectif, qui est la puissance disposant de ces processus dans lesquels l'objectif se supprime dans le contact de ses éléments les uns avec les autres, est lui-même en dehors d'eux et ce qui en eux se conserve, est la ruse de la raison » HEGEL, Encyclo-Logique (1817), \# 160, p.265 ; G6, 127.

17 B. BOURGEOIS,. "La spéculation hégélienne" conf. prononcée à Neuchâtel en 1987, article accessible in Etudes hégéliennes, Raison et décision, Paris, PUF, 1992, p.99. 
mes et de souffrances - à l'une des régions de cette culture conquise longtemps auparavant ». 18

Auschwitz, nous l'avons déjà dit, est ainsi un tel anéantissement que rien ne rattrapera. Il ne s’agit pas ici dans le mal décrit par Hegel uniquement de la souffrance des «instruments » individuels qui feraient au bout du compte advenir quand même la raison, mais aussi du tragique de la raison elle-même contredite au cœur de ses figures dans le devenir humain.

L’histoire, d'un point de vue hégélien, n’est donc pas le paysage où toute défaite apparente (la destruction de la culture) cache toujours une victoire.

\section{3) À l'encontre de tout relativisme conformiste, l'hégélianisme met en place des critères permettant de distinguer un bien et un mal.}

$\underline{\text { Retour à la table des matières }}$

Pour nous permettre de dégager un contraste avec le hégélianisme, évoquons tout d'abord un autre univers philosophique : celui du scepticisme antique. Le scepticisme est abstention. Abstention au niveau théorique, abstention au niveau politique. Le Sceptique ne peut donc intervenir activement dans l'histoire. Il laisse l'histoire passer avec son évanescence. Il laisse Auschwitz se dérouler. Il ne peut être qu'un relativiste conformiste. M. Conche, qui s'est tellement approché du pyrrhonisme que sa manière de penser en est imprégnée, ne peut s'empêcher néanmoins d'en remarquer les limites au niveau politique. Dans un article du $M a-$ gazine littéraire, il déclare ainsi : «Qui oserait ne pas condamner Auschwitz ? Auschwitz signifie l’impossibilité radicale du scepticisme en morale. Et comme la politique, en ce qu'elle doit être, c'est-à-dire comme politique morale, doit rendre impossible un Auschwitz à l'avenir, il faut dire que la politique aussi marque la frontière où s’arrête le scepticisme ». ${ }^{19}$ Le propos est définitif. On ne peut en matière politique s’abstenir d'avancer des critères - ne serait-ce qu’à titre de di-

18 HEGEL, la raison dans l'histoire, p.152.

19 M. CONCHE, «Le scepticisme philosophique et sa limite », in Magazine littéraire N³94, janvier 2001 ; p.20. 
rection générale. Hegel, lui, ne se contente pas d'une abstention face aux mouvements de l'histoire. Il avance bien des critères d'analyse :

« lorsque (...) il est question d'un vrai Etat ou d'un vrai chef d'œuvre (...) Ces ob-jets sont vrais lorsqu'ils sont ce qu'ils doivent être, c'està-dire lorsque leur réalité correspond à leur concept. Ainsi appréhendé, le non-vrai est la même chose que ce que l'on appelle par ailleurs aussi le mauvais. Un mauvais homme n'est pas un vrai homme, c'est-à-dire est un homme qui ne se comporte pas conformément à son concept ou à sa détermination. Sans aucune identité du concept et de la réalité, rien cependant ne peut avoir de consistance. Même ce qui est mauvais et non-vrai n'est que dans la mesure où sa réalité se comporte encore de quelque manière conformément à son concept. Ce qui est entièrement mauvais ou contraire au concept est précisément par là quelque chose qui se désagrège en soi-même ». 20

Hegel aurait, sans doute aucun, qualifié le régime nazi de « mauvais ». Et cela pas seulement parce que rétrospectivement il s'est désagrégé face aux autres nations du monde. La défaite militaire, n'étant qu'un critère « après-coup » n’est pas un critère du tout (nous retomberions dans l'attentisme pragmatique). La notion de durée d'un régime semble en effet un critère flottant : le stalinisme a eu une durée et une stabilité qui n’aurait rien à envier à celles de la démocratie athénienne du Vème siècle av. J.-C.. Mais c'est à partir des principes mêmes d'un régime politique que Hegel se permettrait de le juger. Le régime nazi, ainsi, ne reposait pas sur des principes susceptibles de permettre à l'individu de se penser et de s'accomplir au sein de la totalité. C'est pourquoi il ne pouvait faire sens au niveau de l'histoire, quand bien même eût-il duré mille ans. Nous avons remarqué plus haut que la liberté de l'individu n'était pas la fin de la démarche philosophique hégélienne. Cela est vrai. Non parce que la liberté de l'individu n'a pas d'importance aux yeux du philosophe allemand, mais parce que la liberté individuelle ne pourra pas être fondamentalement garantie sans un horizon plus vaste qui la porte. C'est d'ailleurs la grande ambiguïté de notre modernité occidentale. A ne donner de valeur universelle qu'à ce qui s'arrête à l'individu (c'est le subjectivisme individualiste érigé en fondement universel), se dissout la possibilité même d'une valeur universelle. La valeur de l'individu ne repose-t-elle pas en effet sur ce qui à travers cet individu se joue de plus important que lui ?

20 HEGEL, Encyclo-Logique (1830), add. \# 213, p.615 ; G8 p.424. 


\section{4) Hegel a en réalité indiqué certaines sources du Mal totalitaire.}

Retour à la table des matières

Le danger véritable pour l'homme - Hegel l'a compris - vient moins d'une surdétermination de la vie de l’individu par l’Absolu (qui a lieu de toute façon) que d'une surdétermination de sa vie par l'entendement ${ }^{21}$. En rester à la logique d'entendement pour diriger les hommes mène à ne plus les considérer comme des « particuliers-universels » mais comme simples parties d'un tout (comme des objets). La critique hégélienne à l'encontre de Kant ou de Fichte est la fameuse critique du devoir-être, de la belle âme qui propose un monde meilleur qu'il faudrait accomplir dans un avenir temporellement infini. Nous rappelons une formule hégélienne :

« Saisir et comprendre ce qui est, telle est la tâche de la philosophie, car, ce qui est, c'est la raison. En ce qui concerne l'individu, chacun est le fils de son temps. Il en est de même de la philosophie : elle saisit son temps dans la pensée. Il est aussi insensé de prétendre qu'une philosophie, quelle qu'elle soit, puisse franchir le monde contemporain pour aller au-delà, que de supposer qu'un individu puisse sauter par dessus son temps (...) Si sa théorie va effectivement au-delà, si elle se construit un monde tel qu'il doit être, ce monde existera sans doute, mais seulement dans sa pensée ». 22

21 Nous rappelons que Hegel appellera pensée d'entendement ce qui correspond à la logique classique, celle qui décompose le monde qui nous entoure en divers « objets » (grammaticalement « des sujets ») (exemple : «l'arbre ») et leur donne des attributs (exemple: "vert»). Chez Hegel le dialectique consiste en la reconnaissance de la contradiction au sein d'un objet (et est cette contradiction même). La raison spéculative correspondra en, revanche à une façon radicalement nouvelle de penser le réel. Aucun « objet » n’a de réalité stable. Penser spéculativement c'est montrer comment chaque objet présente une contradiction interne et comment cette contradiction est ce qui amène un autre objet et ainsi de suite jusqu'à l' « objet » total qui est le Système du réel. Le point de vue spéculatif surplombera donc l'ensemble des contradictions et aboutira au réel total qui, seul, n'est pas contradictoire. Le spéculatif est le dépassement du dialectique.

22 HEGEL Principes-philo-droit, Préface, Hoffmeister, p.16 ; trad. p.57. 
Le « monde tel qu'il doit être » n'existera que dans la pensée de la belle âme. Mais le pire arrive quand la belle âme veut faire exister le monde de sa théorie d'entendement dans la réalité. C'est le hégélianisme lu de façon impropre (c'està-dire messianiquement) qui devient alors un danger.

Nous avons vu plus haut la critique formulée par Hegel à l'encontre de Platon et Fichte : ils se sont « empêtrés » à vouloir formuler des conseils concrets immédiatement accessibles à l'entendement en matière de politique. Platon et Fichte ont eu la «chance » de ne pas devenir des hommes politiques (même si Platon a voulu un peu «s'y frotter »). Certains totalitarismes viennent en effet d'hommes pourvus des meilleures intentions du monde - du point de vue de l'entendement. Et sur ce point Hegel tire ici les leçons de son analyse de l' " actualité ». La Révolution française et ses idéaux de liberté - qui parleront néanmoins toujours au cœur de Hegel - est retombée en Terreur car les Révolutionnaires ont cru que la vie concrète des individus (la Sittlichkeit) pouvait être informée de façon immédiate par un principe absolu défini par l'entendement. Ici se trouve le véritable rationalisme dogmatique et son horreur historique. Ici se trouve la source de tous les totalitarismes. Et l'on voit que si Hegel a été accusé d'incarner ce monisme de la pensée générateur de totalitarisme, telle ne doit pas être ce que nous devons lire du hégélianisme. Hegel pointe du doigt l'erreur des révolutionnaires français :

« Il faut regarder seulement comme une sottise des temps modernes, de changer un système d'une éthique corrompue, la constitution politique et la législation liées à elle, sans modifier la religion, d'avoir fait une Révolution sans une Réforme ». 23

Cela signifie très exactement que l'homme n'est pas seulement ni essentiellement la partie d'un tout politique (comme l'affirment les conceptions contractualistes de la société) mais qu'il est spirituellement habité par ce tout, et que sans changement spirituel de l'homme, aucun changement matériel ne peut avoir un sens profond et durable.

Dès lors, il y a parfois plus de vérité pour l'homme dans ce qui au niveau de l'individu paraît opaque à l'entendement que dans ce qui peut être présenté avec clarté et distinction. Hegel nous en donne une illustration concrète :

23 HEGEL, Encyclo-Esprit (1830), \# 552 Remarque, p.338 ; NP, 435. 
« les deux rives d'un fleuve appartiennent typiquement au même pays. La Silésie est le bassin de l’Oder, la Bohême et la Saxe sont la vallée de l'Elbe, l'Egypte celle du Nil. C'est un faux principe qu'ont soutenu les Français pendant les guerres de la révolution, lorsqu'ils prétendaient que les fleuves seraient les frontières naturelles des pays ». 24

Affirmer que les fleuves sont des frontières naturelles c'est affirmer un principe que l' « entendement cartographique » reçoit comme une évidence. Mais bouleverser le réel pour y faire passer de force ce genre d' « idée », c'est exactement ce qui a donné les désastres totalitaires. Au Cambodge, Pol Pot voudra ainsi faire passer de façon immédiate les populations des villes dans les champs et les transformer en populations d'agriculteurs. On connaît l'ampleur de la famine qui s'ensuivit.

Si la protection de l'individu - on l'a évoqué plus haut - n'est pas l'horizon de la politique hégélienne, elle y est nécessairement incluse. Pour le philosophe allemand, il faut naturellement laisser le particulier vivre sa vie de particulier. Il y a un bonheur humain qu'il faut regarder éclore et chercher à préserver (et c'est à travers une attention au droit que la philosophie peut avoir son utilité en ce sens). Hegel ne fait donc pas de l'individu un simple rouage de l'universel. Dans un commentaire oral à son cours sur la philosophie de l'histoire il indique bien le concept de liberté comme critère de discrimination entre les divers Etats :

« la détermination essentielle de la constitution s'énonce dans la proposition suivante : le meilleur Etat est celui dans lequel règne le plus de liberté. Mais la question se pose de savoir d'où la liberté tient sa réalité ». 25

Le « mais » montre à nouveau que le hégélianisme ne saurait se satisfaire d'un horizon réduit au subjectivisme moderne.

Pour comprendre la nette différence entre le hégélianisme et la tendance repérée plus haut dans le discours de Trotski, il faut repérer que l’individu hégélien n’est pas un infirme face à la totalité. L’homme hégélien a accès à la pensée de l'Absolu. Le marxisme, lu dans son obsession pratique, veut en revanche que l'obéissance aveugle au Parti soit au moins pour un temps la clef de voûte de l'efficacité. Mais il y a au bout du compte un mépris foncier pour l'homme chez

24 HEGEL, La raison dans l'histoire, p.227.

25 HEGEL, Commentaire oral à La raison dans l'histoire, p.169. 
ceux qui veulent que l'individu croie, sans repenser les choses. Nous sommes bien loin de l'état d'esprit de Hegel. L'obsession de l'efficacité technique de la théorie est étrangère au philosophe allemand. Tout d'abord l'individu hégélien n'est pas un infirme face à l'histoire. Même s'il ne peut prédire l'histoire, c'est-à-dire savoir à l'avance ce que l'Esprit du Monde sera, c'est toujours lui qui lira le sens de l'histoire. Si dans sa puissance, c'est l'Esprit du Monde qui fait l'histoire, en son sens c'est l'individu philosophe qui en restera le seul lecteur. Ce sens, Hegel ne prétend pas - contrairement aux messianismes politiques postérieurs - le décrypter pour en faire un "catéchisme » vite digéré par des millions de « courroies de transmission ». Hegel est fondamentalement un enseignant - c'est-à-dire quelqu'un qui veut permettre à ses étudiants de repenser eux-mêmes la totalité. Le hégélianisme est étymologiquement encyclopédique, c'est-à-dire qu'il veut permettre à l'élève (l'enfant, le paidos) d'entrer lui aussi dans le cercle (en-kyklô 26) qui se pense lui-même, c'est-à-dire de participer à l'Esprit. L'Encyclopédie c'est l'éducation par le cercle, la montée vers un savoir qui aboutit à son fondement. Son travail est donc patient, obscur même pour ceux qui n'auraient pas la patience de se pencher longuement sur la façon dont le sens fait sens. L'état d'esprit hégélien se lit en revanche clairement dans les derniers mots - mots magnifiques - prononcés devant ses étudiants à la toute fin de son cours sur l'histoire de la philosophie :

« J'ai essayé de dérouler ce cortège des configurations spirituelles de la philosophie dans leur progression en indiquant leur connexion, de le faire défiler devant votre pensée. Cette série est le véritable royaume des esprits, l'unique royaume des esprits qu'il y ait ; - série qui n'est pas une multiplicité, qui ne demeure pas non plus une série au sens de succession, mais qui précisément dans la connaissance de soi-même fait de soi des moments de l'unique esprit, (fait de soi) le seul et même esprit présent. Et ce long cortège d'esprits, ce sont les pulsations singulières dont il se sert dans sa vie ; ils sont l'organisme de notre substance (...) j'ai eu plaisir à avoir été avec vous dans cette communauté

26 Nous rappelons néanmoins qu'il s'agit d'une étymologie apparaissant dans le latin de la Renaissance (ENCYCLOPAEDIA) (Budé 1508), mais qu'elle a été construite d'après le grec enkyklopaideia (compris comme « instruction embrassant le cercle des connaissances ») à la suite d'une erreur de lecture d'un manuscrit de Plutarque, qui parlait d'egkuklios paideia («instruction complète »). 
de vie spirituelle, et non seulement d’y avoir été, mais je l'espère, d'avoir noué avec vous un lien spirituel : puisse ce lien demeurer entre nous ! Je vous souhaite de très bien vivre». 27

\section{Copyright Bertrand QUENTIN}

27 HEGEL, Leçons-hist-philo T7, « Résultat », p. 2117 ; G19, p. 691. 